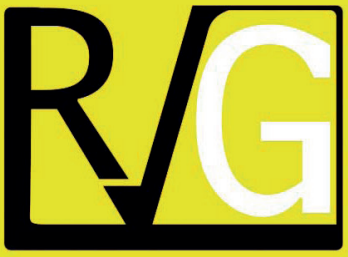

Julio - Septiembre, 2021

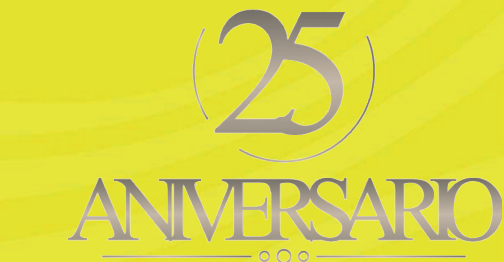

ANMERSARO
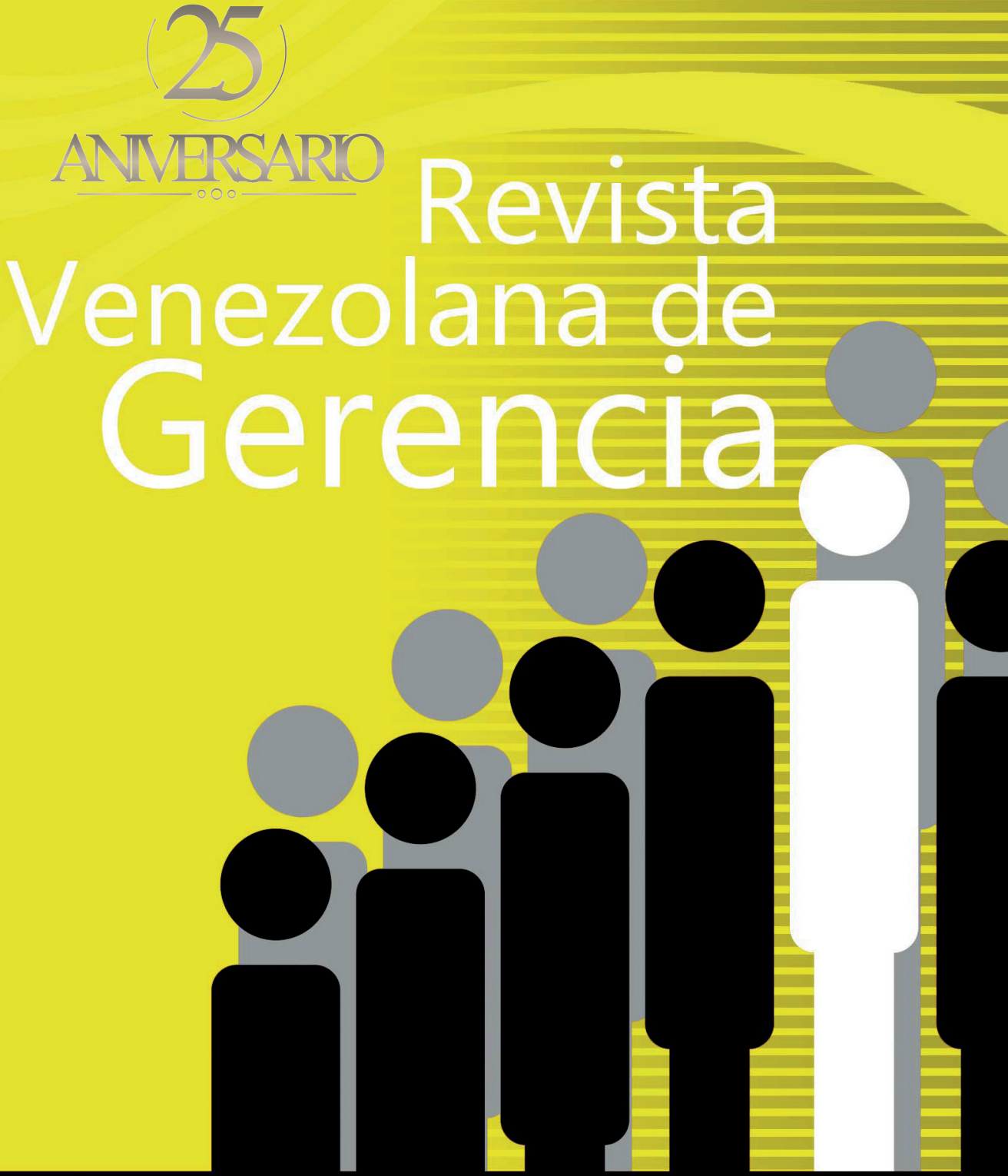

UNIVERSIDAD DEL ZULIA (LUZ)

Facultad de Ciencias Económicas y Sociales Centro de Estudios de la Empresa

ISSN 1315-99

Esta obra está bajo una licencia de Creative Comm Reconocimiento-NoComercial-Compartirlgual 3.0 Unpo http://creativecommons.org/licenses/by-nc-sa/3.0/deed.es 
COMO CITAR: Abad-Segura, E., González-Zamar, M.-D., y LópezMeneses, E. (2021). Gestión financiera para la toma decisiones en las universidades: Producción científica y tendencias en investigación. Revista Venezolana de Gerencia, 26(95), 705-722. https://doi.org/10.52080/ rvgluz.27.95.17
Universidad del Zulia (LUZ)

Revista Venezolana de Gerencia (RVG)

Año 26 No. 95 Julio-Septiembre 2021, 705-722

ISSN 1315-9984 / e-ISSN 2477-9423

\title{
Gestión financiera para la toma decisiones en las universidades: producción científica y tendencias en investigación
}

\author{
Abad-Segura, Emilio* \\ González-Zamar, Mariana-Daniela** \\ López-Meneses, Eloy***
}

\section{Resumen}

Las instituciones de educación superior se enfrentan, en la actualidad, a nuevos desafíos basados en encontrar el modo de dar respuestas válidas a las demandas que impone la sociedad, y que se suponen cambios para estas instituciones universitarias. Este estudio tiene como objetivo analizar la producción científica y las tendencias de investigación sobre la gestión financiera para la toma decisiones en las universidades. Para esto, se aplicaron técnicas bibliométricas a una muestra de 1.306 artículos de la base de datos Scopus, durante el período 1990-2019. La finalidad fue reconocer las disciplinas y la evolución de estas áreas de interés, además de identificar las redes por coautoría de los principales autores, organizaciones y países, y la red de palabras clave por coocurrencia, mediante la aplicación de técnicas de visualización. Los resultados obtenidos suponen un complemento al conocimiento y permiten fundamentar el

Recibido: 01-02-21

Aceptado: 15-06-21

* Doctor en Ciencias Económicas y Empresariales, Universidad de Almería-España; Máster en Contabilidad y Finanzas Corporativas. Profesor Docente e Investigador, Universidad de Almería-España. Email: eas297@ ual.es, ORCID: https://orcid.org/0000-0001-8624-103X

** Doctor en Ciencias de la Educación, Universidad de Almería-España; Máster en Investigación y Evaluación Didáctica en el Aula para el Desarrollo Profesional Docente. Profesora Docente e Investigadora, Universidad de Almería-España. Email: mgz857@ual.es, ORCID: https://orcid.org/0000-0003-1187-8970

*** Doctor en Ciencias de la Educación, Universidad de Sevilla-España; Profesor titular, Universidad Pablo de Olavide-España. Director del grupo de investigación EDUINNOVAGOGÍAC (HUM-971); Coordinador del Grupo Internacional Innovagogía(; Email: elopmen@upo.es, ORCID: https://orcid.org/0000-0003-0741$\underline{5367}$ 
proceso de la toma de decisiones. Se ha observado que las tendencias generales en la investigación sobre la gestión financiera para la toma decisiones en las instituciones de educación superior a nivel mundial han seguido una tendencia ascendente y se han estabilizado en óptimos índices de publicación en los últimos años.

Palabras clave: producción científica; tendencias en investigación; gestión financiera; toma de decisiones; universidades.

\title{
Financial management for decision-making in higher education institutions: Research trends
}

\begin{abstract}
Higher education institutions are currently facing new challenges based on finding a way to provide valid responses to the demands imposed by society, and that changes are assumed for these university institutions. This study aims to analyse scientific production and research trends on financial management for decision-making in universities. For this, bibliometric techniques were applied to a sample of 1306 articles from the Scopus database, during the period 1990-2019. The purpose was to recognize the disciplines and the evolution of these areas of interest, in addition to identifying the networks by coauthorship of the main authors, organizations and countries, and the keyword network by co-occurrence, through the application of visualization techniques. The results obtained are a complement to the knowledge and allow to base the decision-making process. It has been observed that the general trends in research on financial management for decision-making in higher education institutions worldwide have followed an upward trend and have stabilized at optimal publication rates in recent years.
\end{abstract}

Keywords: scientific production; research trends; financial management; decisionmaking; universities.

\section{Introducción}

La actividad de una organización se produce en un entorno cambiante y globalizado, que le obliga a mantenerse en un perenne y sistemático proceso de búsqueda y renovación (GangaContreras, 2017; Obstfeld, 2020;
Ganga-Contreras et al. 2020), en el que la concentración de capital arriesga la pluralidad de medios (Mayorga, 2012). Por esta razón, su administración debe estar capacitada para pensar estratégicamente la organización e identificar, evaluar y aprovechar los distintos escenarios para generar valor 
en ella (Ganga-Contreras, 2013; Rincón y Chaparro, 2018; Oliveiros y Vargas, 2017).

Las finanzas respaldan la estructura de una empresa, dan sustento a las operaciones y permiten el crecimiento de cualquier negocio, aunque en ocasiones, como en el resto de los recursos, pueden llegar a ser limitadas por factores monetarios, humanos $u$ otras contingencias (Herrero et al. 2017). Por tanto, es clave para una organización administrar las finanzas de manera eficiente, es decir, elaborar estrategias financieras (Hussaini, 2019) adaptadas a cada caso (Prado et al. 2018; López-Pérez y Olvera-Lobo, 2019).

A nivel organizacional se deben tomar decisiones en un entorno crecientemente competitivo, la mayoría de las ocasiones, sin opción de error (Ganga-Contreras et al. 2015). Esas decisiones, estratégicas y operativas, principalmente, se basan en elementos cuantitativos que, a partir de modelos de decisión, permiten obtener información que facilitan las determinaciones (Sax y Andersen, 2019; Sánchez et al. 2015). Por estos motivos, el gestor debe identificar los elementos clave que serán de utilidad para examinar los criterios a partir de los que se tomarán las decisiones, basadas en el análisis de la información disponible.

En esta línea, la gestión financiera en una organización se refiere a la función encargada de la rentabilidad, los gastos, el efectivo y el crédito, de modo que ésta pueda tener los medios para llevar a cabo su objetivo de la manera más satisfactoria posible, es decir, maximizar el valor de la organización para los accionistas (Ramos-Monge et al. 2019; Obstfeld, 2020).
La gestión financiera examina el tratamiento y el análisis del dinero y las inversiones de una organización para ayudar a tomar decisiones comerciales (Sandoval-Estupiñán, 2020). No obstante, las decisiones de gestión financiera son el presupuesto de capital, la estructura de capital y la gestión del capital de trabajo (Yan, 2019; AbelloRomero, Mancilla y Viancos, 2019) y humano (Pérez y Romero, 2009) incluyendo la revalorización de este (Castañón, 2018; Iglesias et al. 2017) y el valor asociado a la innovación (Salgado, 2014).

Asimismo, la gestión financiera ayuda a administrar los recursos de la organización y lleva a realizar la planificación financiera de la empresa. En este contexto, los directores financieros son los profesionales especializados que dependen directamente de la alta dirección (Rajnoha et al. 2019). En relación con la gestión, son los encargados de planificar y organizar la estrategia económica, y de controlar las operaciones que se lleven a cabo en la compañía. Igualmente, en la gestión financiera se superponen dos áreas: gerenciales y corporativas (Binda y Balbastre-Benavent, 2013; Chirinos, 2017). La primera se ocupa de la importancia gerencial de las técnicas económicas; mientras que la segunda, atiende al capital a largo plazo de las grandes corporaciones (Zaid et al. 2020).

La gestión financiera se dirige a la toma óptima de decisiones, es decir, al proceso de identificar y elegir entre distintas alternativas para reportar beneficios a la organización (SandovalEstupiñán et al. 2020) con atención a las consideraciones de condiciones laborales en sectores culturales (Oliva, 2017) y a las nuevas realidades 
comunicacionales (Sierra, 2001). Cada proceso de toma de decisiones produce una elección final, que puede o no impulsar la acción.

Así, en relación con las decisiones operativas, la organización deberá gestionar con eficacia las medidas administrativas: control de la contabilidad, gestión de impuestos, política de compras y de selección de proveedores, control de costes, gestión de la tesorería, medidas de atención al cliente (Rodríguez y Ramírez, 2019; Ramos-Monge et al. 2019) o gestión de ayudas públicas (Lara et al. 2018; Hasper et al. 2017).

Este estudio está contextualizado en las instituciones cuya actividad se focaliza en la educación superior, es decir, aquella que incluye la docencia, la investigación, el trabajo aplicado y las actividades de servicios sociales de las universidades (Abello-Romero, et al. 2019; López-Meneses et al. 2020). Se enmarca en los principios de la teoría de la agencia (Jensen y Meckling, 1976), como medida de representación organizacional con validez legal donde se separa la propiedad de la organización de su gestión. Es clave indicar que cada una de las partes busca la maximización de su utilidad, y que el poder de decisión da lugar a la aparición de intereses no comunes.

La principal motivación de este trabajo es analizar la producción científica y las tendencias de investigación sobre la gestión financiera para la toma decisiones en las universidades, cuya creciente complejidad quedó atestiguada por González (2014) y por la irrupción entre las variables de las consideraciones de inversión en las Tecnologías de la Información y la Comunicación (TIC), como queda de manifiesto en Celaya et al. (2020), Cuesta et al. (2020) o Catalina et al. (2019) y los nuevos medios de financiamiento, como los descritos por Felipe et al. (2018).

En la revisión de la literatura realizada, se han encontrado estudios que vinculan la toma de decisiones realizadas por las empresas en su actividad con los métodos cuantitativos. De este modo, la pregunta de investigación consiste en determinar cómo ha evolucionado la producción científica, cómo colaboran los principales actores impulsores de este campo de investigación, y cuáles son las líneas de investigación en esta temática.

La principal limitación encontrada en este estudio es la de comprender si la evolución de las tendencias de investigación se debe a cambios normativos, a exigencias de los grupos de interés, o a demandas sociales.

El objetivo principal de este estudio es analizar la producción científica y las tendencias de investigación sobre la gestión financiera para la toma decisiones en las instituciones de educación superior a nivel global. Para obtener respuestas a las preguntas de investigación, se analizó una muestra de 1.306 artículos extraídos de la base de datos Scopus, desde 1.990 a 2.019. Esta revisión utiliza el método bibliométrico para sintetizar la base de conocimiento sobre la investigación de la gestión financiera para la toma decisiones en las instituciones de educación superior. Los resultados muestran las publicaciones en esta temática, permitiendo así identificar a los principales agentes impulsores (autores, organizaciones de investigación y países) y las tendencias de investigación. 


\section{Consideraciones metodológicas de la investigación}

La bibliometría se define como la aplicación de métodos matemáticos y estadísticos a revistas, libros y otros medios de comunicación (Pritchard, 1969). La finalidad de esta metodología es identificar y analizar los componentes principales dentro de un determinado campo de investigación específico, de modo que en este estudio se refiere a la producción científica sobre la gestión financiera de las instituciones universitarias y la toma de decisiones.

Además, permite presentar la evolución del interés por la temática objeto de estudio, al manifestar los autores, países, revistas y palabras clave más relevantes durante los últimos años (Vinkler, 2010), se plantea mostrar una visión general de la dinámica de la investigación sobre la gestión financiera para la toma decisiones en las instituciones de educación superior, desde 1990 a 2019. Con este propósito, se han utilizado herramientas matemáticas, estadísticas y de mapeo. Los trabajos de investigación de una disciplina científica se pueden clasificar a través del análisis bibliográfico de acuerdo con su información, como la suma de documentos, número de citas, número de referencias utilizadas, palabras clave, organizaciones, países, autores, índice h, entre otros (DelgadoVázquez et al. 2019; Abad-Segura y González-Zamar, 2020).

Para seleccionar la muestra de artículos, se realizó una búsqueda en la base de datos Scopus. En ésta se incluyeron los siguientes términos, en inglés, aunando la producción de este campo de investigación: "management", "finance", "decision-making", "organization", "higher education institution" y "university". La elección de estos términos atiende a los de mayor valor descriptivo y representatividad, de acuerdo con la revisión de la literatura realizada. Así, se incluyen los campos de título, resumen y palabras clave. La cobertura temporal corresponde el período comprendido entre 1990 y 2019 , esto es, 30 años.

La muestra final incluyó 1.306 artículos, donde únicamente se incluyeron los documentos de las áreas afines a la investigación, es decir, "Business, Management and Accounting" y "Economics, Econometrics and Finance". Se ha tenido en cuenta una amplia diversidad de variables a analizar para cada registro, como el año de publicación, la revista, el área temática, el autor y coautores del trabajo, la afiliación institucional de los autores, el país de afiliación y las palabras clave que definen el documento, como se ha aplicado en otras publicaciones con éxito (López et al. 2015; Abad-Segura et al. 2020b).

A partir de las técnicas bibliométricas se pueden crear, visualizar y explorar los mapas bibliográficos. En estos, los enlaces muestran las conexiones entre los distintos elementos. En este trabajo se muestran los enlaces basados en los métodos de coautoría, para autores, organizaciones y países, y de coocurrencia, para las palabras clave (Abad-Segura et al. 2020a). Así, los enlaces y los artículos forman una red bibliográfica conjunta (White y McCain, 1998). Para analizar y visualizar la literatura científica se utilizó el software VOSviewer (Van Eck y Waltman, 2010). 
Gestión financiera para la toma decisiones en las instituciones de educación superior: Análisis de tendencias

\section{Gestión financiera para la toma decisiones: Resultados y discusión de la producción científica de las instituciones de educación superior}

Esta sección presenta los resultados y discusión sobre el tema de investigación relacionado con la producción científica de las instituciones de educación superior, referidas a la gestión financiera para la toma decisiones. También se atienden las relaciones de cooperación establecidas entre los principales actores impulsores de este tema, y el análisis de las palabras clave que permite identificar las líneas de investigación desarrolladas en este campo, como las de Sánchez-Marín et al. (2019) o Zamora et al. (2018) en lo semántico y de Melicherová et al. (2021) en cuanto a la fórmula empleada.

El gráfico 1 muestra la evolución de la producción científica a nivel internacional en el campo de investigación de la gestión financiera para la toma decisiones en las instituciones de educación superior, en el período 1990-2019. Se observa, de los 1.306 documentos en el período de 30 años estudiado, 634 se han publicado en los últimos 5 años (2015-2019), es decir, el $48,55 \%$ del total de las contribuciones. En el último año analizado, 2019, se han publicado 169 artículos (12,94\%). De modo que se manifiesta el interés por el estudio de esta área de investigación a nivel internacional, con una publicación creciente desde el inicio y exponencial desde el 2000. Los artículos analizados se han escrito en 13 idiomas diferentes, con 1.283 en inglés $(98,24 \%)$.

\section{Gráfico 1 \\ Evolución de la producción científica (1990-2019)}

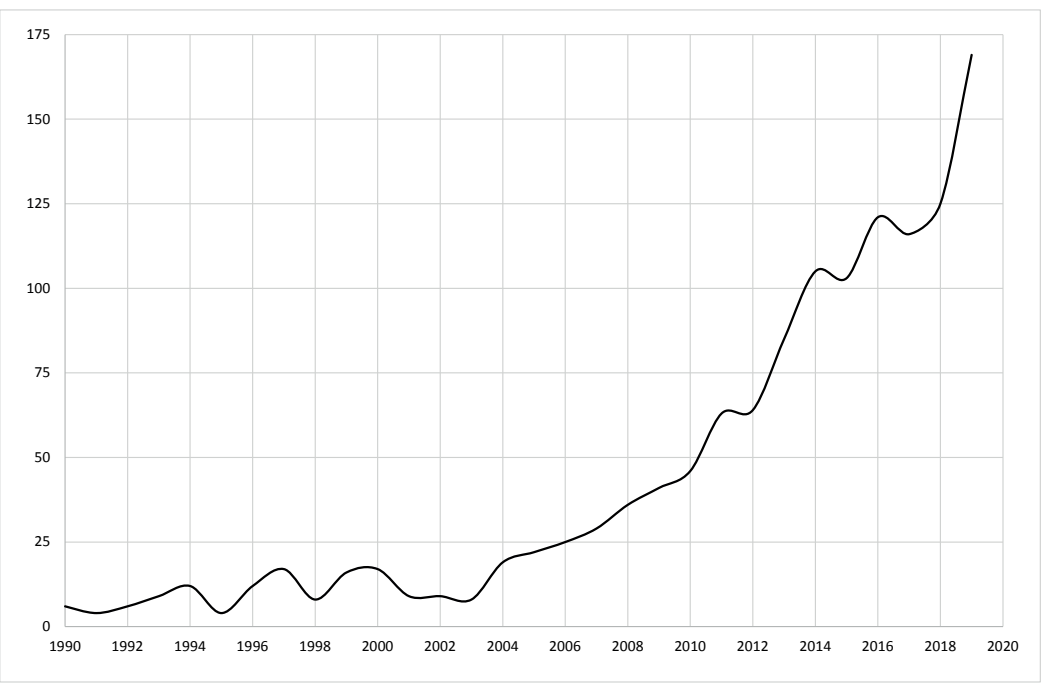

Fuente: Elaboración propia (2020). 
El primer artículo publicado en este período data de 1990, con el título An econometric model of a public university's income and enrollments, publicado en la revista International Journal of Economic Behavior y Organization, por los autores Hoenack, Stephen A., y Daniel J. Pierro, ambos afiliados a la Universidad de Minnesota Twin Cities (Minneapolis, Estados Unidos), haciendo relevante la temática desde hace más de un siglo.

Asimismo, el artículo más citado, publicado en 2008 por la revista Journal of Accounting Research, lleva por título International accounting standards and accounting quality, y fue redactado por los autores Barth, et al. (2008) (Stanford University, Palo Alto, Estados Unidos), Wayne R. Landsman y Mark H. Lang (ambos afiliado a la Universidad de North Carolina en Chapel Hill, Chapel Hill, Estados Unidos).

En relación a las principales redes de cooperación, estas se analizan considerando los distintos agentes impulsores de esta temática de estudio (autores, instituciones y países). La muestra de 1.306 artículos fue escrita por 2.842 autores. En este sentido los autores que más han publicado sobre la temática de estudio han sido: Doost, R.K., con 6 artículos, además de Jooste, L. y Scapens, R.W., con 4 artículos cada uno. De este modo, se puede observar un gran número de autores los cuales de forma armónica mantienen un número de publicaciones, evitando la hegemonía de unos sobre otros.

El gráfico 2 muestra la red de colaboración científica basada en la coautoría de artículos sobre gestión financiera para la toma decisiones en las instituciones de educación superior. Los autores se asociaron, según la herramienta VOSviewer, en 3 grupos. El grupo 1, de color rosa, lo encabeza Benková E. y se asocia con Dobrovič J., Gallo P., Balogová B., Mihalčová B., Urbański M. y Čabinová V. El grupo 2, de color verde, está liderado por Štefko R. y se asocia con Jenčová S., Litavcová E., Mat'ovčíková D. y Vašaničová $P$. Finalmente, el grupo 3, de color rojo, está liderado por Rajnoha R. y se vincula con Krajčík V., Lesníková P. y Merková M.

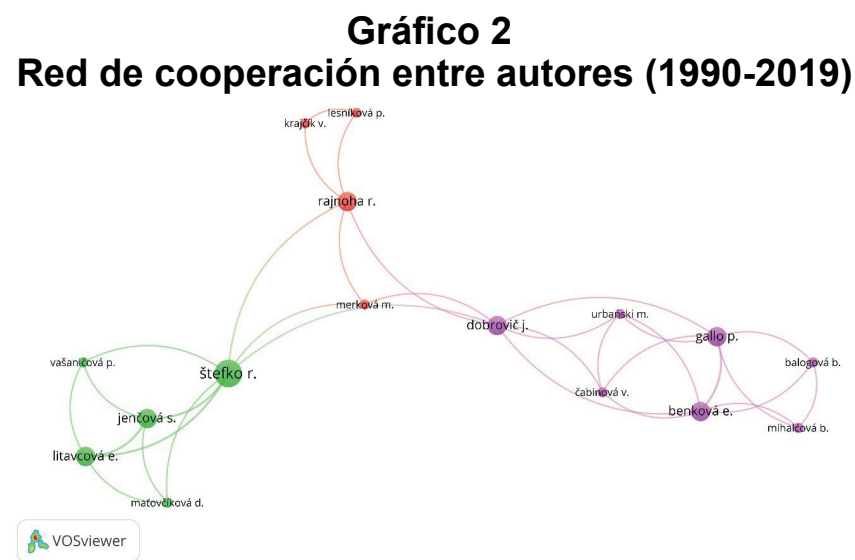

Fuente: elaboración propia con el software VOSviewer (2020) 
Abad-Segura, Emilio; González-Zamar, Mariana-Daniela; López-Meneses, Eloy; Luque de la Rosa, Antonio

Gestión financiera para la toma decisiones en las instituciones de educación superior: Análisis de tendencias

La publicación de las contribuciones se ha distribuido entre 2.236 instituciones a nivel global. Las más productivas han sido la National Bureau of Economic Research y la Vilniaus Gedimino Technikos Universitetas, con 16 artículos publicados cada una; la University of Sydney y la University of Chicago, con 13; y la University of Illinois en Urbana-Champaign y la Vysoká Škola Ekonomická v Praze, con 12.

El gráfico 3 muestra la red de colaboración científica entre las instituciones según la coautoría de documentos sobre la temática de estudio, asociándose en 3 grupos. El grupo 1 (color rosa) está liderado por Ku Leuven (Leuven, Bélgica), y se asocia con la Faculty of Business and Economics
(Dortmund University, Vogelpothsweg, Alemania), Iza (Bonn, Alemania), y Zew (Mannheim, Alemania).

El grupo 2 (color verde) está encabezado por Case Western Reserve University, (Cleveland, Estados Unidos), y se asocia con Boston University (Boston, Estados Unidos) y el Department of Marketing and Policy Studies, de Weatherhead School of Management - Case Western Reserve University (Cleveland, Estados Unidos). Por último, el grupo 3 (color rojo) está liderado por Hasselt University (Diepenbeek, Bélgica), se asocia con Ku Leuven (Brussels, Bélgica), y Maastricht University (Maastricht, Países Bajos), gráfico 3.

\section{Gráfico 3 \\ Red de cooperación entre organizaciones (1990-2019)}

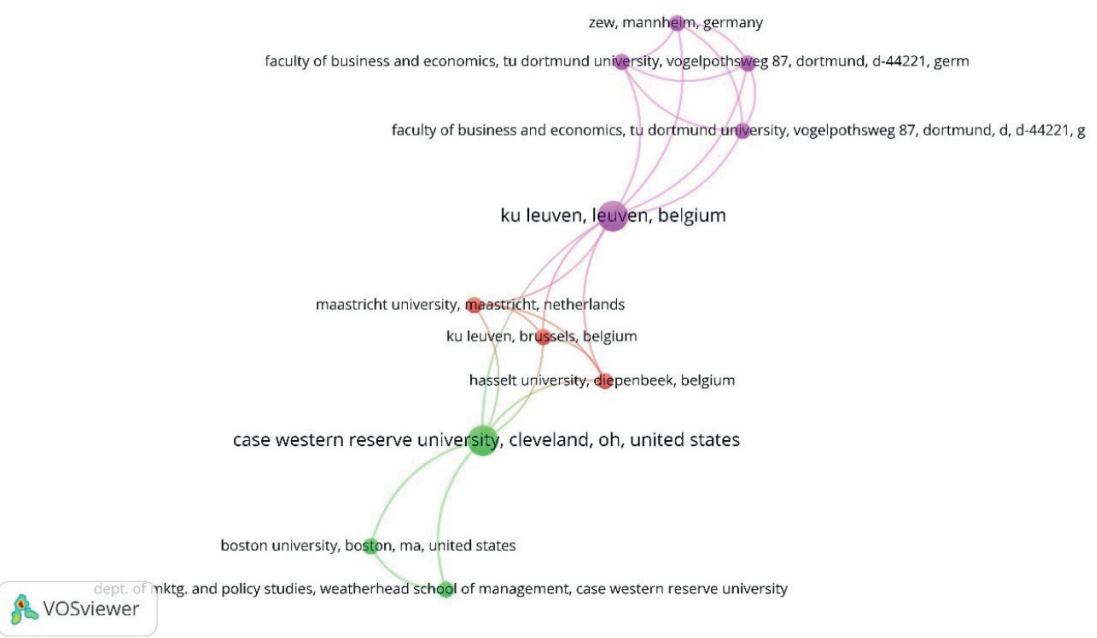

Fuente: elaboración propia con el software VOSviewer (2020) 
Asimismo, al desarrollo de este campo de investigación han contribuido 86 países. Los diez más prolíficos ha sido Estados Unidos, con 382 artículos publicados, Reino Unido (122), Australia (79), República Checa (51), Canadá (43), Taiwán (40), España (39), China (37), Polonia (36) y Malasia (35).

El gráfico 4 representa el mapa de colaboración entre los principales países basado en la coautoría de sus autores. El software VOSviewer ha detectado la asociación según 6 grupos. El grupo 1 (color rosa), el más numeroso, está encabezado por Estados Unidos y se asocia con Reino Unido, Canadá, España, Alemania, Italia, Países Bajos, Francia, Suiza, Bélgica, Portugal, Suecia, Brasil y Dinamarca. El grupo 2 (verde) está liderado por Republica Checa y se asocia con Taiwán, China, Polonia, Eslovaquia, Sudáfrica, Indonesia, Rusia, Nueva Zelanda, Turquía, Hungría y Filipinas.

\section{Gráfico 4 \\ Red de cooperación entre países (1990-2019)}

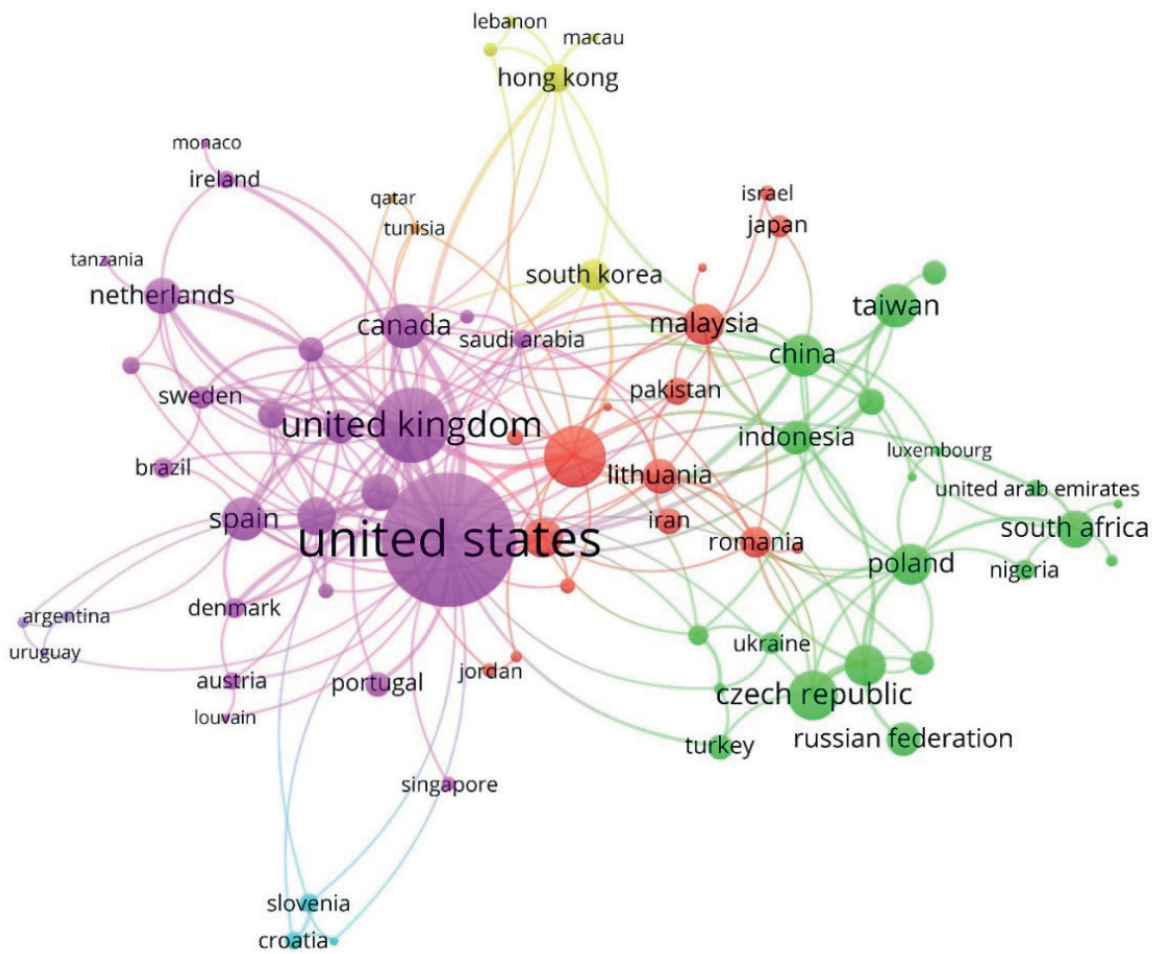

Fuente: elaboración propia con el software VOSviewer (2020) 
Gestión financiera para la toma decisiones en las instituciones de educación superior: Análisis de tendencias

El grupo 3 (rojo) está encabezado por Australia y se asocia con Malasia, India, Lituania, Rumania, Pakistán, Irán, Japón, Finlandia, Bahréin, Israel, Jordania, Bulgaria y Irak. El grupo 4 (amarillo) está liderado por Corea del Sur y se vincula con Hong Kong, México, Líbano y Macao. El grupo 5 (violeta), el menos numeroso, está encabezado por Argentina y se asocia con Colombia y Uruguay. Por último, el grupo 6 (azul) está encabezado por Eslovenia y se asocia con Croacia, Bosnia y Herzegovina, Túnez y Qatar. Los países que se han sumado a la contribución de la temática de estudio más recientemente han sido: en 2017: Rusia, Tailandia, Irak, Sri Lanka, Bangladesh, Macao, y Bosnia y Herzegovina; en 2018: Kazajstán, Brunei y Luxemburgo; y en 2019: Mónaco.
El análisis del conjunto de palabras clave de un tema específico de indagación permite conocer las principales líneas de investigación que se desarrollan por los principales agentes que la impulsan. Con el propósito de observar la evolución de las tendencias en este campo, se realizó un análisis de las 4.013 palabras clave utilizadas en los 1.306 artículos.

El gráfico 5 presenta el mapa de red para las palabras clave de los artículos analizados sobre la gestión financiera para la toma decisiones en las instituciones de educación superior. El tamaño del círculo representa el número de artículos en los que aparece cada palabra clave, y el color informa del grupo en el que se incluye la palabra clave en función del número de apariciones conjuntas.

\section{Gráfico 5 Red de palabras clave (1990-2019)}

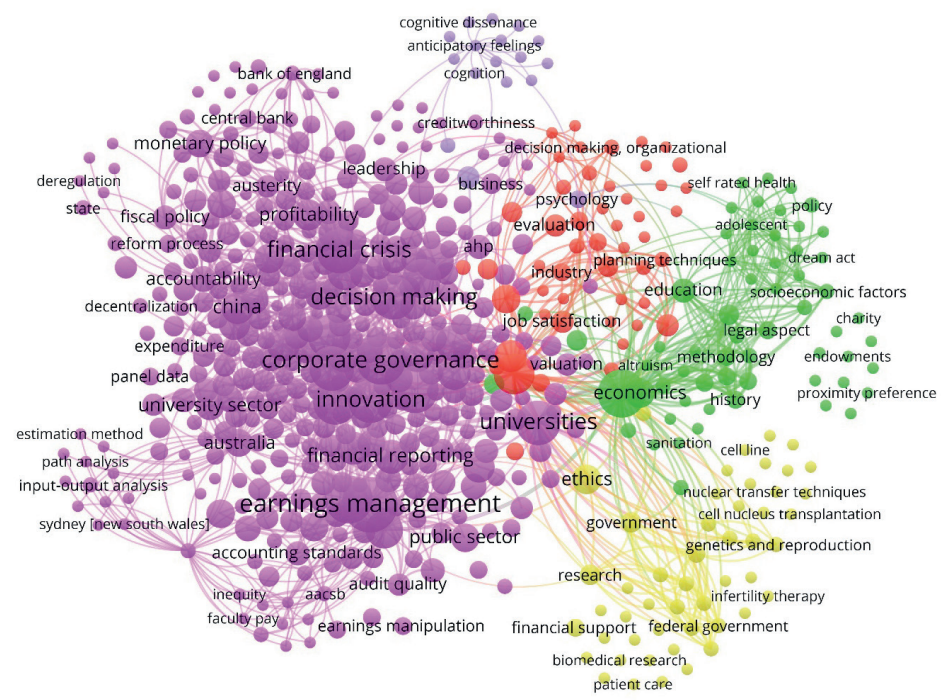

Fuente: elaboración propia con el software VOSviewer (2020) 
Se han identificado 5 grupos principales, representando los distintos puntos de vista sobre la investigación de este campo de estudio. Las principales palabras clave asociadas a cada grupo son: earnings management (gestión de ingresos), financial management (gestión financiera), entrepreneurship (emprendimiento), ethics (ética), y organizational culture (cultura organizacional), en torno a las cuales se vinculan el resto de los términos de las publicaciones sobre este tema de investigación.

El grupo 1 (rosa) está liderado por el término gestión de ingresos. Se asocia con: gobierno corporativo, educación superior, gestión de riesgos, innovación, finanzas, crisis financiera, administración, universidades, toma de decisiones, rendimiento financiero, inversión, contabilidad, estudiantes y sistema financiero. El grupo 2 (verde) está encabezado por el término gestión financiera y se asocia con Ciencias Económicas, educación, responsabilidad social, aspecto legal, protección del medio ambiente, recaudación de fondos, política, bienestar social, factores socioeconómicos, impacto en el bienestar, éxito académico, altruismo, caridad y estudiante universitario.

En el grupo 3 (rojo) destaca el término emprendimiento y se vincula con organización, evaluación, satisfacción laboral, organización y gestión, aprendizaje, motivación, creatividad, industria, objetivos organizacionales, capital de riesgo, innovación organizacional, gestión de personal, técnicas de planificación, trabajo en equipo y logro académico. El grupo 4 (amarillo) está liderado por el término ética y se asocia con gobierno, soporte financiero, regulación gubernamental, control social, revisión ética, oficina de integridad científica, sector privado, opinión pública, mala conducta científica, control social, informal. El grupo 5 (violeta) está liderado por el término cultura organizacional $y$ se asocia a términos tales como: pensamiento grupal, mercado, caídas del mercado, exuberancia del mercado, moral, moralidad, exceso de seguridad, burbujas especulativas y activos tóxicos.

Así, las líneas de investigación se han dirigido durante este período a añadir contribuciones que aporten herramientas de valor agregado y estrategias a la gerencia financiera para optimizar la toma de decisiones de las instituciones de educación superior, tales como la gestión del riesgo, estrategia financiera óptima, el análisis de las relaciones entre variables financieras, o las implicaciones políticas y educativas en las finanzas de las instituciones (Sandoval-Estupiñán, 2020).

Asimismo, estos componentes se centran en examinar la estrategia de precios variables, a fin de maximizar los ingresos o las ganancias de un recurso fijo y limitado en el tiempo. Además, la actividad científica se ha focalizado en el análisis de la gestión del rendimiento que implica el control estratégico del inventario de las instituciones de educación superior (Abello-Romero et al. 2019).

La Tabla 1 muestra las 20 principales palabras clave, según el número de artículos y de enlaces, referidos a la conexión de coocurrencia entre dos palabras clave. En este sentido, los términos "gestión de ingresos" y "gobierno corporativo" son los más frecuentes, esto es, en 46 y 34 artículos respectivamente. Por otro lado, los términos "gestión financiera" y "universidades" destacan en relación con el número de enlaces, con 171 y 134 , respectivamente. 
Abad-Segura, Emilio; González-Zamar, Mariana-Daniela; López-Meneses, Eloy; Luque de la Rosa, Antonio

Gestión financiera para la toma decisiones en las instituciones de educación superior: Análisis de tendencias

\section{Tabla 1 \\ Palabras clave (1990-2019)}

\begin{tabular}{|c|c|c|c|c|}
\hline Posición & Palabra clave (inglés) & Palabra clave (español) & Artículos & Enlaces \\
\hline 1 & Earnings Management & Gestión de Ingresos & 46 & 40 \\
\hline 2 & Corporate Governance & Gobierno Corporativo & 34 & 36 \\
\hline 3 & Financial Management & Gestión Financiera & 32 & 171 \\
\hline 4 & Higher Education & Educación Superior & 32 & 69 \\
\hline 5 & Risk Management & Gestión de Riesgos & 31 & 26 \\
\hline 6 & Innovation & Innovación & 29 & 64 \\
\hline 7 & Financial Crisis & Crisis Financiera & 27 & 116 \\
\hline 8 & Finance & Finanzas & 27 & 112 \\
\hline 9 & Management & Administración & 27 & 64 \\
\hline 10 & Universities & Universidades & 27 & 134 \\
\hline 11 & Decision Making & Toma de Decisiones & 26 & 86 \\
\hline 12 & Performance & Actuación & 24 & 35 \\
\hline 13 & Financial Performance & Rendimiento Financiero & 20 & 23 \\
\hline 14 & Investment & Inversión & 19 & 115 \\
\hline 15 & Accounting & Contabilidad & 18 & 33 \\
\hline 16 & Students & Estudiantes & 17 & 32 \\
\hline 17 & Financial System & Sistema Financiero & 16 & 98 \\
\hline 18 & $\begin{array}{l}\text { Corporate Social Re- } \\
\text { sponsibility }\end{array}$ & $\begin{array}{c}\text { Responsabilidad Social } \\
\text { Corporativa }\end{array}$ & 15 & 28 \\
\hline 19 & Financial Reporting & Informes Financieros & 14 & 16 \\
\hline 20 & Governance & Gobernancia & 13 & 23 \\
\hline
\end{tabular}

Fuente: Elaboración propia (2020)

Por otro lado, es un campo de investigación con cierta madurez, puesto que son pocos los términos incorporados a la temática de investigación en los últimos años. Las más pioneras han tenido mayor influencia, actuando como referencia para las que han ido surgiendo con posterioridad. La existencia de 5 grupos claramente diferenciados permite entender como la gestión financiera para la toma decisiones en las instituciones de educación superior
Así, las últimas palabras clave añadidas por año fueron, en 2018: manipulación de ganancias, autoinforme, acreditación, falta de equidad, inversión, revisión por pares, métodos cuantitativos y modelos de valoración; y en 2019: desregulación, gasto público, provisión social, gestión de deuda soberana, y papel del estado.

\section{Conclusiones}

El número de artículos por año 
durante el período analizado, 1990-2019, se ha incrementado, especialmente, en los últimos cinco años, con 634 artículos, que representando el $48,55 \%$ del total de las contribuciones sobre esta temática. En el último año analizado, 2019, se publicaron 169 artículos (12,94\%) en 13 idiomas diferentes, con 1.283 en inglés $(98,24 \%)$.

En esta temática de estudio los autores más productivos son: Doost, R.K., Jooste, L. y Scapens, R.W; y se asocian en 3 grupos. Por otro lado, las instituciones de investigación más productivas han sido la National Bureau of Economic Research y la Vilniaus Gedimino Technikos Universitetas, con 16 artículos publicados cada una; y se asocian en 3 grupos. Asimismo, los países más prolíficos han sido Estados Unidos, Reino Unido, Australia, República Checa y Canadá; y se han asociado en 6 grupos de colaboración.

Las principales palabras clave detectadas en el registro de publicaciones fueron earnings management, corporate governance, financial management, higher education y risk management. En el período analizado se han identificado 5 principales líneas de investigación en este campo de estudio, que contribuyen con artículos dedicados a: earnings management (gestión de ingresos), financial management (gestión financiera), entrepreneurship (emprendimiento), ethics (ética), y organizational culture (cultura organizacional).

El análisis aplicado de este estudio presentar algunas limitaciones metodológicas. En este sentido, la base de datos consultada podría afectar la selección de documentos, aunque es necesario tener en cuenta que Scopus incluye la mayoría de los artículos incluidos en otras bases de datos (Arencibia y Peralta, 2020). Además, cabe señalar que una consulta de búsqueda diferente puede dar resultados diferentes, y que, en este trabajo, el estudio cuantitativo realizado en este estudio se podría completar con un análisis cualitativo.

En la actualidad, las instituciones de educación superior se enfrentan a nuevos retos basados en encontrar el modo de dar respuesta eficiente y eficaz a las demandas sociales, convirtiéndose en un desafío para estas instituciones universitarias. En esta línea, la educación superior ha dejado de ser un bien de consumo vinculado a una minoría de la población, convirtiéndose en un importante factor de desempeño económico de los diversos impulsores del conocimiento y en una expectativa para el individuo en la sociedad contemporánea. Por lo tanto, la investigación de este campo debe continuar desarrollándose en nuevas líneas que exploren diferentes aspectos y fomenten la optimización del proceso de toma de decisiones de las instituciones de educación superior.

Finalmente, es necesario destacar que las tendencias generales en la investigación sobre la gestión financiera para la toma decisiones en las instituciones de educación superior a nivel mundial han seguido una tendencia ascendente y se han estabilizado en óptimos índices de publicación en los últimos años. Los resultados obtenidos suponen un complemento al conocimiento y permiten fundamentar el proceso de la toma de decisiones.

\section{Referencias bibliográficas}

Abad-Segura, E., González-Zamar, M. D., Infante-Moro, J. C., y Ruipérez García, G. (2020a). 
Abad-Segura, Emilio; González-Zamar, Mariana-Daniela; López-Meneses, Eloy; Luque de la Rosa, Antonio

Gestión financiera para la toma decisiones en las instituciones de educación superior: Análisis de tendencias

Sustainable Management of Digital Transformation in Higher Education: Global Research Trends. Sustainability, 12(5), 2107. https:// doi.org/10.3390/su12052107

Abad-Segura, E., González-Zamar, M.-D., López-Meneses, E., y Vázquez-Cano, E. (2020b). Financial Technology: Review of Trends, Approaches and Management. Mathematics, 8(6), 951. https://doi. org/10.3390/math8060951

Abad-Segura, E., y González-Zamar, M.D. (2020). Global Research Trends in Financial Transactions. Mathematics, 8(4), 614. https://doi.org/10.3390/ $\underline{\text { math8040614 }}$

Abello-Romero, J., Mancilla, C., y Viancos, P. (2019). Diversidad del derecho a la informacion y su efecto divulgativo en universidades de América Latina. Utopía y Praxis Latinoamericana, 24(S4), 47-64. https://produccioncientificaluz.org/ index.php/utopia/article/view/29922

Arencibia-Jorge, R., y Peralta-González, M.J. (2020). Recomendaciones sobre el uso de Scopus para el estudio de las Ciencias de la Información en América Latina. Revista Iberoamericana de Medición y Comunicación de la Ciencia, 1(1). https://doi.org/10.47909/ijsmc.07

Barth, M. E., Landsman, W., y Lang, M. (2008) International accounting standards and accounting quality. Journal of accounting research, 46(3), 467-498. https://onlinelibrary. wiley.com/doi/full/10.1111/j.1475679X.2008.00287.x

Binda, N. U.; y Balbastre-Benavent, F. (2013). Investigación cuantitativa e investigación cualitativa: buscando las ventajas de las diferentes metodologías de investigación. Revista de Ciencias económicas, 31(2), 179-187. https://revistas.ucr. ac.cr/index.php/economicas/article/ view/12730

Castañón, N. (2018). Elementos que motivan al usuario a obtener certificación de "cursos en línea" en Educación Superior. Revista de ciencias de la Comunicación e Información, 23(1), 1-14. https://doi. org/10.35742/rcci.2018.23(1).1-14

Catalina-García, B., López de AyalaLópez, M. C., y Martínez Pastor, E. (2019). Usos comunicativos de las nuevas tecnologías entre los menores. Percepción de sus profesores sobre oportunidades $y$ riesgos digitales. Mediaciones Sociales, 18, 43-57. https://doi. org/10.5209/meso.64311

Celaya, I., Ramírez-Montoya, M. S., Naval, C. y Arbués, E. (2020). Usos del podcast para fines educativos. Mapeo sistemático de la literatura en WoS y Scopus (2014-2019). Revista Latina de Comunicación Social, 77, 179-201. https://doi.org/10.4185/ RLCS-2020-1454

Chirinos, M.E. (2017). La Gerencia como fenómeno y la Complejidad. Sistemas y conexiones. Utopía y Praxis Latinoamericana, 22(78), 119133. https://produccioncientificaluz. org/index.php/utopia/article/ view/22641/22448

Cuesta Cambra, U., Cuesta Díaz, V., Martínez Martínez, L. y Niño González, J. I. (2020). Smartphone: en comunicación, algo más que una adicción. Revista Latina de Comunicación Social, 75, 367-381. www.doi.org/10.4185/RLCS-20201431

Delgado-Vázquez, Á. M., Cano, E. V., Montoro, M. R. B., y Meneses, E. L. (2019). Análisis bibliométrico del impacto de la investigación educativa en diversidad funcional y competencia digital. Web of Science 
y Scopus. Aula abierta, 48(2), 147156. https://dialnet.unirioja.es/ servlet/articulo?codigo $=6931049$

Felipe, A., Caldevilla, D., Rodríguez, J. y Del Valle Mejías, M. (2018). Crowdfunding: comunicación del mecenazgo 2.0: filantropía digital en las relaciones públicas bidireccionales. Revista de ciencias de la comunicación e información, 23(1), 29-41. http://doi.org/10.35742/ rcci.2018.23(1).29-41

Ganga-Contreras, F. (2013). Gerencia latinoamericana en las actuales plataformas organizacionales: Una mirada preliminar y sinóptica. Revista Venezolana de Gerencia, 18(64), 711722. https://produccioncientificaluz. org/index.php/rvg/article/view/11172

Ganga-Contreras, F., Ramos, E., Leal, A., y Pérez, K. (2015). Administración estratégica: Aplicación del cuadro de mando integral (CMI) a una organización no gubernamental. Revista de Ciencias Sociales, 21(1), 136-159. https:/l produccioncientificaluz.org/index. php/rcs/article/view/25712

Ganga-Contreras, F. (2017). El flipper burocrático en las universidades. Revista Interciencia, 42(1), 5862. https://www.redalyc.org/ jatsRepo/339/33949290010/html/ index.html

Ganga-Contreras, F., Sáez, W., Calderón, A., Calderón, Á. y Rodríguez-Ponce, E. (2020). Principales rankings académicos internacionales: el caso de Chile. Ensaio: Avaliação e Políticas Públicas em Educação, 28(107), 407-434. https://dx.doi.org/10.1590/ s0104-40362019002701964

González, B. (2014). Complejidad y sistematización de la evaluación de la educación superior en México. Revista de Comunicación de la SEECI, 33, 01-12. https://doi.

\section{org/10.15198/seeci.2014.33.1-12}

Hasper, J., Correa, J., Benjumea, M. y Valencia, A. (2017). Tendencias en la investigación sobre gestión del riesgo empresarial: un análisis bibliométrico. Revista Venezolana de Gerencia, 22, 506-524. https://www. produccioncientificaluz.org/index. php/rvg/article/view/23036

Herrero-Gutiérrez, F-J., MartínezVallvey, F., Tapia-Frade, A., ReyGarcía, P. y Cabezuelo-Lorenzo, F. (2017). Transparencia en el sector público a través de salas de prensa online. Gestión de recursos colectivos y su información en webs municipales. El profesional de la información, 26(3), 421-429. https:// doi.org/10.3145/epi.2017.may.08

Hoenack, S. A., y Daniel, J. P. (1990). An econometric model of a public university's income and enrollments. Journal of Economic Behavior y Organization, 14(3), 403-423. https:// www.sciencedirect.com/science/ article/abs/pii/016726819090067N

Hussaini, A. (2019). Organizational management through strategic planning and financial practices: An empirical assessment from business firms of Kuwait. Management Science Letters, 9(5), 713-726. https://doi. org/10.5267/j.msl.2019.2.001

Iglesias, E., Pastor, E., y Rondón, L. M. (2017). Mediación como profesión emergente: actualidad formativa desde la Educación Superior. Mediaciones Sociales, 16, 135-153. https://doi.org/10.5209/MESO.58113

Jensen, M. C., y Meckling, W. H. (1976). Theory of the firm: Managerial behavior, agency costs and ownership structure. Journal of financial economics, 3(4), 305-360. https:// www.sciencedirect.com/science/ article/pii/0304405X7690026X 
Abad-Segura, Emilio; González-Zamar, Mariana-Daniela; López-Meneses, Eloy; Luque de la Rosa, Antonio

Gestión financiera para la toma decisiones en las instituciones de educación superior: Análisis de tendencias

Lara, M., García, N., y Bueno, T. (2018). La innovación docente en los estudios de Comunicación: análisis documental de los proyectos financiados por las universidades públicas madrileñas. CIC. Cuadernos de información y comunicación, 23, 143-156. https://doi.org/10.5209/ CIYC.60911

López-Meneses, E., Vázquez-Cano, E., y Román Graván, P. (2015). Análisis e implicaciones del impacto del movimiento MOOC en la comunidad científica: JCR y Scopus (201013). Comunicar, XXII (44), 73-80. https://www.redalyc.org/articulo. oa?id=15832806008

López-Meneses, E., Vázquez-Cano, E., González-Zamar, M. D., y AbadSegura, E. (2020). Socioeconomic effects in cyberbullying: global research trends in the educational context. International journal of environmental research and public health, 17(12), 4369. https://doi. org/10.3390/ijerph17124369

López-Pérez, Lourdes; Olvera-Lobo, María-Dolores (2019). Participación digital del público en la ciencia de excelencia española: análisis de los proyectos financiados por el European Research Council. El profesional de la información, 28(1), 1-10. https://doi.org//10.3145/ epi.2019.ene.06

Mayorga, A.J. (2012). Reflexiones sobre democracia y medios de comunicación. Perspectivas de la comunicación, 5(2) 158-168. https:// bit.ly/3weXqJr

Melicherová, M., Ondrišová, M. y Šušol, J. (2021). Bibliometrics versus altmetrics: Researchers' attitudes in Slovakia. Revista Iberoamericana de Medición y Comunicación de la Ciencia. 1(1). https://doi. org/10.47909/ijsmc.11
Obstfeld, M. (2020). Globalization Cycles. Italian Economic Journal, 6(1), 1-12. https://link.springer.com/ article/10.1007/s40797-020-00121-4

Oliva Abarca, J.E. (2017). El trabajo cultural: estudio local sobre las condiciones laborales en tres subsectores culturales. Perspectivas de la comunicación, 10(2), 143-170. http://revistas.ufro.cl/ojs/index.php/ perspectivas/article/view/824

Oliveros, J. A., \& Vargas, N. (2017). Diagnóstico financiero de la Pequeña y Mediana Industria aplicando gerencia de valor. Revista Venezolana De Gerencia, 22(79), 486-505. https://doi.org/10.37960/ revista.v22i79.23035

Pérez, M.J. y Romero, M. (2009). La gestión de las personas en el nuevo entorno empresarial de la comunicación. VIVAT Academia. Revista de comunicación, 109, 21-43. https://doi.org/10.15178/ va.2009.109.21-43

Pritchard, A. (1969). Statistical bibliography or bibliometrics. Journal of documentation, 25(4), 348-349. https://bit.ly/2UiOE1w

Rajnoha, R., Lesnikova, P., Stefko, R., Schmidtova, J., y Formanek, I. (2019). Transformations in strategic business planning in the context of sustainability and business goals setting. Transformations in Business y Economics, 18(2), 44-66. http:// www.transformations.knf.vu. It/47/ rp47.pdf

Ramos-Monge, E. L., Llinàs-Audet, X., y Barrena-Martínez, J. (2019). Catalysts of university social responsibility into strategic planning by thematic analysis and deductive coding. International journal of entrepreneurship and innovation management, 23(4), 327-355. http:// 
www.transformations.knf.vu.It/47/ rp47.pdf

Rincón, I.C. y Chaparro, J.E. (2018). Creación de valor en empresas privadas del sector salud en el Departamento de Córdoba (Colombia). Opción, 34, 1256-1278. https://produccioncientificaluz.org/ index.php/opcion/article/view/23975

Rodríguez, Y. A., y Ramírez, M. A. (2019). Estrategia de integración universidad-entidad laboral para la capacitación de los cuadros en la gestión de la información con las Tecnologías de la Información y las Comunicaciones. Revista Referencia Pedagógica, 7(1), 27-38.

Sánchez-Marín, F. J., Parra-Meroño, M. C., y Peña-Acuña, B. (2019). Experiencias de trabajo cooperativo en la educación superior. Percepciones sobre su contribución al desarrollo de la competencia social. Vivat Academia. Revista de Comunicación, 147, 87-108. http:// doi.org/10.15178/va.2019.147.87$\underline{108}$

Sandoval-Estupiñán, L. Y., Pineda-Baéz, C., Bernal-Luque, R., y Quiroga, C. (2020). Los retos del director escolar novel: formación inicial y liderazgo. Revista Complutense de Educación, 31(1), 117-127. https://revistas. ucm.es/index.php/RCED/article/ view/61919

Sánchez, J. M., Bolaños, M. V., Magallón, R., y Caffarena, V. A. (2015). El papel de las tecnologías cívicas en la redefinición de la esfera pública. Historia y Comunicación Social, 20(2), 483-498. https://doi. org/10.5209/rev HICS.2015.v20. $\underline{\mathrm{n} 2.51396}$

Salgado, M. C., González, M. J., y Zamarra, M. de las M. (2014). Innovación y aplicación tecnológica en el ámbito de la Educación Superior universitaria. El empleo de los blogs en las universidades españolas. Historia y Comunicación Social, 18, 613-625. https://doi.org/10.5209/rev HICS.2013.v18.44353

Sax, J., y Andersen, T. J. (2019). Making risk management strategic: Integrating enterprise risk management with strategic planning. European Management Review, 16(3), 719-740. https://onlinelibrary. wiley.com/doi/abs/10.1111/ emre.12185

Sierra, F. (2001). La educación superior y los sistemas multimedia de interacción simbólica. CIC. Cuadernos de información $y$ comunicación, (6), 333. https:// revistas.ucm.es/index.php/CIYC/ article/view/CIYC0101110333A

Van Eck, N.J., y Waltman, L. (2010). Software survey: VOSviewer, a computer program for bibliometric mapping. Scientometrics, 84 , 523-538. https://link.springer.com/ article/10.1007/s11192-009-0146-3

Vinkler, P. (2010). Indicators are the essence of scientometrics and bibliometrics. Scientometrics, 85(3), 861-866. https://link.springer.com/ article/10.1007/s11192-010-0159-y

White, H. D. y McCain, K. W. (1998). Visualizing a discipline: An author co-citation analysis of information science, 1972-1995. Journal of the American society for information science, 49(4), 327-355. https://bit. Iy/3wglNWo

Yan, S. H. I. (2019). Research on the Optimizing Path of University Budgetary Planning Based on Strategic Perspective. Shanghai Management Science, 21(3). https:// papers.ssrn.com/sol3/papers. cfm?abstract id $=3021984$

Zaid, M. A., Wang, M., Adib, M., 
Abad-Segura, Emilio; González-Zamar, Mariana-Daniela; López-Meneses, Eloy; Luque de la Rosa, Antonio

Gestión financiera para la toma decisiones en las instituciones de educación superior: Análisis de tendencias

Sahyouni, A., y Abuhijleh, S. T. (2020). Boardroom nationality and gender diversity: Implications for corporate sustainability performance. Journal of Cleaner Production, 251, 119652. https://www.sciencedirect. com/science/article/abs/pii/ $\underline{\text { S0959652619345226 }}$
Zamora, R., Falcón, M., y M. Tapia Pullas, P. (2018). Implicación del modelo de aprendizaje basado en proyectos colaborativos en el contexto universitario. Revista de Comunicación de la SEECl, 46, 01-11. $\quad$ http://doi.org/10.15198/ seeci.2018.46.01-11 